\title{
THE EXPLICIT LAPLACE TRANSFORM FOR THE WISHART PROCESS
}

\author{
ALESSANDRO GNOATTO,${ }^{*}$ LMU München \\ MARTINO GRASSELLI,** Università di Padova, Devinci Finance Lab, Pole Universitaire \\ Léonard de Vinci and Quanta Finanza S.R.L.
}

\begin{abstract}
We derive the explicit formula for the joint Laplace transform of the Wishart process and its time integral, which extends the original approach of Bru (1991). We compare our methodology with the alternative results given by the variation-of-constants method, the linearization of the matrix Riccati ordinary differential equation, and the Runge-Kutta algorithm. The new formula turns out to be fast and accurate.
\end{abstract}

Keywords: Affine process; Wishart process; ordinary differential equation; Laplace transform

2010 Mathematics Subject Classification: Primary 65C30

Secondary $60 \mathrm{H} 35 ; 91 \mathrm{~B} 70$

\section{Introduction}

In this paper we propose an analytical approach to the computation of the moment generating function for the Wishart process introduced in [5], as an extension of square Bessel processes (see [35] and [37]) to the matrix case. Wishart processes belong to the class of affine processes and they generalise the notion of the positive factor in so far as they are defined on the set of positive-semidefinite real $d \times d$ matrices, denoted by $S_{d}^{+}$. Given a filtered probability space $\left(\Omega, \mathcal{F}, \mathcal{F}_{t}, \mathbb{P}\right)$ satisfying the usual assumptions and a $d \times d$ matrix Brownian motion $B$ (i.e. a matrix whose entries are independent Brownian motions under $\mathbb{P}$ ), a Wishart process on $S_{d}^{+}$is governed by the stochastic differential equation (SDE)

$$
\mathrm{d} S_{t}=\sqrt{S_{t}} \mathrm{~d} B_{t} Q+Q^{\top} \mathrm{d} B_{t}^{\top} \sqrt{S_{t}}+\left(M S_{t}+S_{t} M^{\top}+b\right) \mathrm{d} t, \quad S_{0} \in S_{d}^{+}, t \geq 0,
$$

where $Q \in \mathrm{GL}_{d}$ (the set of invertible real $d \times d$ matrices), $M \in M_{d}$ (the set of real $d \times d$ matrices) with all eigenvalues on the negative half-plane in order to ensure stationarity, and where the matrix $b$ satisfies $b \succeq(d-1) Q^{\top} Q$, that is, $b-(d-1) Q^{\top} Q \in S_{d}^{+}$. In the literature, the constant drift term is often of the more restrictive form $b=\alpha Q^{\top} Q$ for $\alpha \geq d-1$. In the case where the (Gindikin) real parameter $\alpha$ satisfies $\alpha \geq d+1$, the process takes values in the interior of $S_{d}^{+}$, denoted by $S_{d}^{++}$, in analogy with the Feller condition for the scalar case. In the dynamics above $\sqrt{S_{t}}$ denotes the square root in the matrix sense. Existence and uniqueness results for the solution of (1) may be found in [5] under parametric restrictions and in [32] in full generality. We denote by $\operatorname{WIS}_{d}\left(S_{0}, b, M, Q\right)$ the law of the Wishart process $\left(S_{t}\right)_{t \geq 0}$.

Received 12 December 2012; revision received 13 August 2013.

* Postal address: Mathematisches Institut, LMU München, Theresienstrasse 39, D-80333 München, Germany.

Email address: gnoatto@mathematik.uni-muenchen.de

** Postal address: Dipartimento di Matematica, Università degli Studi di Padova, Via Trieste 63, 35121 Padova, Italy.

Email address: grassell@math.unipd.it 
The starting point of the analysis was the square of a matrix Brownian motion $S_{t}=B_{t}^{\top} B_{t}$, while the generalization to the particular dynamics (1) followed by looking at squares of matrix Ornstein-Uhlenbeck processes (see [5]).

Bru [5] proved many interesting properties of this process, such as noncollision of the eigenvalues (when $\alpha \geq d+1$ under parametric restrictions) and the additivity property shared with square Bessel processes. Moreover, she computed the Laplace transform of the Wishart process and its integral (the matrix Cameron-Martin formula using her terminology), which plays a central role in the applications:

$$
\mathbb{E}_{S_{0}}^{\mathbb{P}}\left[\exp \left\{-\operatorname{tr}\left[w S_{t}+\int_{0}^{t} v S_{s} \mathrm{~d} s\right]\right\}\right] .
$$

Here $\operatorname{tr}[\cdot]$ denotes the trace operator, and $w$ and $v$ are symmetric matrices for which (2) is finite. Bru found an explicit formula for (2) (Formula (4.7) of [5]) under the assumption that the symmetric diffusion matrix $Q$ and the mean reversion matrix $M$ commute.

Positive (semi)definite matrices arise in finance in a natural way and the nice analytical properties of affine processes on $S_{d}^{+}$opened the door to new interesting affine models that allow for nontrivial correlations among positive factors, a feature which is precluded in classic (linear) state space domains like $\mathbb{R}_{\geq 0}^{n} \times \mathbb{R}^{m}$ (see [18]). Not surprisingly, the last years have witnessed the birth of a whole branch of literature on applications of affine processes on $S_{d}^{+}$. The first proposals were formulated in [20]-[23] both in discrete and continuous time. Applications to multifactor volatility and stochastic correlation can be found in [4], [6], [7], [12], [13], [14], [15], and [16] both in option pricing and portfolio management. These contributions consider the case of continuous-path Wishart processes. As far as jump processes on $S_{d}^{+}$are concerned, we recall the proposals in [3], [33], and [34]. Cuchiero et al. [11] and Leippold and Trojani [28] considered jump-diffusion models in this class, while Grasselli and Tebaldi [24] investigated processes lying in the more general symmetric cones state space domain, including the interior of the cone $S_{d}^{+}$(see also the recent developements in [10]).

The main contribution of this paper consists in relaxing the commutativity assumption made in [5], and proving that it is possible to explicitly characterize the joint distribution of the Wishart process and its time integral for a general class of (not even symmetric) mean-reversion and diffusion matrices satisfying the assumptions above with a general constant drift term $b$. The proof of our general Cameron-Martin formula is in line with that of Theorem 2" of [5] and we will provide a step-by-step derivation. The study of transform formulae for affine processes on $S_{d}^{+}$is also the topic of [26], where results concerning Wishart bridges are also provided.

The paper is organized as follows. In Section 2 we prove our main result, which extends the original approach by Bru. In Section 3 we recall some other existing methods which have been employed in the literature for the computation of the Laplace transform: the variation-ofconstants, linearization, and Runge-Kutta methods. The first two methods provide analytical solutions, so they should be considered as competitors of our new methodology. We show that the variation-of-constants method is unfeasible for real-life computations: hence, the truly analytic competitor is the linearization procedure. After that, we present some applications of our methodology to various settings: a multifactor stochastic volatility model, a stochastic correlation model, and a short-rate model. Finally, we present a new approach for the computation of a solution to the algebraic Riccati equation. 


\section{The matrix Cameron-Martin formula}

\subsection{The Wishart process from the point of view of affine processes}

Before we introduce our result, we would like to report some notation and terminology from [11]. Let us recall first the definition of an affine process.

Definition 1. A Markov process $S$ on $S_{d}^{+}$is called affine if it is stochastically continuous and its Laplace transform has exponential-affine dependence on the initial state, i.e. the following equation holds for all $t \geq 0$ and $u \in S_{d}^{+}$:

$$
\mathbb{E}\left[\mathrm{e}^{-\operatorname{tr}\left[u S_{t}\right]}\right]=\int_{S_{d}^{+}} \mathrm{e}^{-\operatorname{tr}[u \xi]} p_{t}(x, \mathrm{~d} \xi)=\exp \left\{-\phi(t, u)-\operatorname{tr}\left[\psi(t, u) S_{0}\right]\right\}
$$

for some functions $\phi: \mathbb{R}_{+} \times S_{d}^{+} \rightarrow \mathbb{R}_{+}$and $\psi: \mathbb{R}_{+} \times S_{d}^{+} \rightarrow S_{d}^{+}$.

In [11], a complete characterization of affine processes on $S_{d}^{+}$is provided in terms of the socalled admissible parameter set (see Definition 2.3 of [11]), which constitutes the affine analogue of a Lévy triplet. The Wishart process with dynamics (1) is a conservative pure diffusion affine process with admissible parameter set $(\alpha, b, B(x), 0,0,0,0)$, where $B(x)=M x+x M^{\top}$ and $\alpha=Q^{\top} Q$. Since the process is affine, it is possible to reduce the Kolmogorov partial differential equation (PDE) associated to the computation of (2) to a nonlinear (matrix Riccati) ordinary differential equation (ODE).

Proposition 1. ([11].) Let $S_{t} \in \mathrm{WIS}_{d}\left(S_{0}, b, M, Q\right)$ be the Wishart process defined by (1). Then

$$
\mathbb{E}_{S_{0}}^{\mathbb{P}}\left[\exp \left\{-\operatorname{tr}\left[w S_{t}+\int_{0}^{t} v S_{s} \mathrm{~d} s\right]\right\}\right]=\exp \left\{-\phi(t)-\operatorname{tr}\left[\psi(t) S_{0}\right]\right\},
$$

where the functions $\psi$ and $\phi$ satisfy the following system of ODEs:

$$
\begin{gathered}
\frac{\mathrm{d} \psi}{\mathrm{d} t}=\psi M+M^{\top} \psi-2 \psi Q^{\top} Q \psi+v, \quad \psi(0)=w, \\
\frac{\mathrm{d} \phi}{\mathrm{d} t}=\operatorname{tr}[b \psi(t)], \quad \phi(0)=0 .
\end{gathered}
$$

\subsection{Statement of the result}

In this section we proceed to prove the main result of this paper. We report a formula completely in line with the matrix Cameron-Martin formula given in [5].

Theorem 1. Let $S \in \mathrm{WIS}_{d}\left(S_{0}, b, M, Q\right)$ be the Wishart process solving (1), assume that

$$
M^{\top}\left(Q^{\top} Q\right)^{-1}=\left(Q^{\top} Q\right)^{-1} M,
$$

let $b \succeq(d+1) Q^{\top} Q$, and define the set of convergence of the Laplace transform as

$$
\mathscr{D}_{t}=\left\{w, v \in S_{d}: \mathbb{E}_{S_{0}}^{\mathbb{P}}\left[\exp \left\{-\operatorname{tr}\left[w S_{t}+\int_{0}^{t} v S_{s} \mathrm{~d} s\right]\right\}\right]<+\infty\right\} .
$$

Then, for all $u, v \in \mathscr{D}_{t}$, the joint moment generating function of the process and its integral is given by

$$
\mathbb{E}_{s_{0}}^{\mathbb{P}}\left[\exp \left\{-\operatorname{tr}\left[w S_{t}+\int_{0}^{t} v S_{s} \mathrm{~d} s\right]\right\}\right]=\exp \left\{-\phi(t)-\operatorname{tr}\left[\psi(t) s_{0}\right]\right\},
$$


where the functions $\phi$ and $\psi$ are given by

$$
\begin{aligned}
\psi(t)= & \frac{\left(Q^{\top} Q\right)^{-1} M}{2}-\frac{Q^{-1} \sqrt{\bar{v}} k(t)\left(Q^{\top}\right)^{-1}}{2} \\
\phi(t)= & \operatorname{tr}\left[b \frac{\left(Q^{\top} Q\right)^{-1} M}{2}\right] t \\
& +\frac{1}{2} \operatorname{tr}\left[\left(Q^{\top}\right)^{-1} b(Q)^{-1} \log \left(\sqrt{\bar{v}}^{-1}(\sqrt{\bar{v}} \cosh (\sqrt{\bar{v}} t)+\bar{w} \sinh (\sqrt{\bar{v}} t))\right)\right],
\end{aligned}
$$

with $k(t)$ given by

$$
k(t)=-(\sqrt{\bar{v}} \cosh (\sqrt{\bar{v}} t)+\bar{w} \sinh (\sqrt{\bar{v}} t))^{-1}(\sqrt{\bar{v}} \sinh (\sqrt{\bar{v}} t)+\bar{w} \cosh (\sqrt{\bar{v}} t))
$$

and $\bar{v}, \bar{w}$ defined as

$$
\bar{v}=Q\left(2 v+M^{\top} Q^{-1}\left(Q^{\top}\right)^{-1} M\right) Q^{\top}, \quad \bar{w}=Q\left(2 w-\left(Q^{\top} Q\right)^{-1} M\right) Q^{\top} .
$$

Moreover, the set where the Laplace transform is regular contains at least the area defined by

$$
\begin{aligned}
v & \succ-M^{\top}\left(2 Q^{\top} Q\right)^{-1} M, \\
w & \succeq\left(2 Q^{\top} Q\right)^{-1} M-Q^{-1} \sqrt{\bar{v}}\left(2 Q^{\top}\right)^{-1} .
\end{aligned}
$$

Remark 1. The derivation of Theorem 1 involves a change of probability measure that will be illustrated in the sequel. This change of measure introduces a lack of symmetry which does not allow us to derive a fully general formula. However, under assumption (5), we are able to span a large class of processes. In fact, equality (5) requires the symmetry of a matrix: this involves $d(d-1) / 2$ linear equalities in $d^{2}$ variables; therefore, if we fix the parameters of the matrix $\left(Q^{T} Q\right)^{-1}$ and consider the constraints on the parameters of $M$, we obtain $d^{2}-d(d-1) / 2=d(d+1) / 2$ degrees of freedom for choosing the matrix $M$.

In the two-dimensional case, let

$$
\left(Q^{\top} Q\right)^{-1}=\left(\begin{array}{ll}
a & b \\
b & c
\end{array}\right), \quad M=\left(\begin{array}{ll}
x & y \\
z & t
\end{array}\right) .
$$

Then condition (5) can be expressed as

$$
b x+c z=a y+t b,
$$

meaning that we can span a large class of parameters, thus going far beyond the commutativity assumption $Q M=M Q$ for $Q \in S_{d}$ and $M \in S_{d}^{-}$as in [5].

Remark 2. Conditions (6) and (7) give explicit parameter constraints in order to ensure the finiteness of the Laplace transform. If they are not satisfied then the Laplace transform is regular only up to a (possibly finite) explosion time. Note that conditions (6) and (7) extend the usual assumptions $v, w \in S_{d}^{+}$as in [11].

\subsection{Proof of Theorem 1}

We will prove the theorem in several steps. We first consider a simple Wishart process with $M=0$ and $Q=I_{d}$, defined under a measure $\tilde{\mathbb{P}}$ equivalent to $\mathbb{P}$. In the second step we introduce the volatility matrix $Q$, using an invariance result. Finally, we will prove the extension for the full process by relying on a measure change from $\tilde{\mathbb{P}}$ to $\mathbb{P}$. Under this last measure, the Wishart process will be defined by the dynamics (1). 
As a starting point, we fix a probability measure $\tilde{\mathbb{P}}$ such that $\tilde{\mathbb{P}} \approx \mathbb{P}$. Under the measure $\tilde{\mathbb{P}}$, we consider a matrix Brownian motion $\hat{B}=\left(\hat{B}_{t}\right)_{t \geq 0}$, which allows us to define the process $\Sigma_{t} \in \mathrm{WIS}_{d}\left(S_{0}, \tilde{b}, 0, I_{d}\right)$, i.e. a process that solves the matrix SDE

$$
\mathrm{d} \Sigma_{t}=\sqrt{\Sigma_{t}} \mathrm{~d} \hat{B}_{t}+\mathrm{d} \hat{B}_{t}^{\top} \sqrt{\Sigma_{t}}+\tilde{b} \mathrm{~d} t, \quad \Sigma_{0} \in S_{d}^{+},
$$

where the drift term $\tilde{b}$ satisfies the condition

$$
\tilde{b}-(d+1) I_{d} \in S_{d}^{+} .
$$

For this process, relying on [5] and [35], we are able to calculate the Cameron-Martin formula. For the sake of completeness, we report the result in [5], which constitutes an extension of the methodology introduced in [35]. The result was proved for the restrictive drift $\alpha I_{d}$, but we will extend it to the general drift by looking to the system of Riccati ODEs.

Proposition 2. ([5], Proposition 5, p. 742.) If $\boldsymbol{\Phi}: \mathbb{R}_{+} \rightarrow S_{d}^{+}$is continuous, constant on $[t, \infty)$, and such that its right derivative (in the distribution sense) $\Phi_{d}^{\prime}: \mathbb{R}_{+} \rightarrow S_{d}^{-}$is continuous, with $\boldsymbol{\Phi}_{d}(0)=I_{d}$ and $\boldsymbol{\Phi}_{d}^{\prime}(t)=0$, then, for every Wishart process $\Sigma_{t} \in \mathrm{WIS}_{d}\left(\Sigma_{0}, \alpha, 0, I_{d}\right)$, we have

$$
\mathbb{E}\left[\exp \left\{-\frac{1}{2} \operatorname{tr}\left[\int_{0}^{t} \boldsymbol{\Phi}_{d}^{\prime \prime}(s) \boldsymbol{\Phi}_{d}^{-1}(s) \Sigma_{s} \mathrm{~d} s\right]\right\}\right]=\left(\operatorname{det} \boldsymbol{\Phi}_{d}(t)\right)^{\alpha / 2} \exp \left\{\frac{1}{2} \operatorname{tr}\left[\Sigma_{0} \boldsymbol{\Phi}_{d}^{+}(0)\right]\right\},
$$

where

$$
\boldsymbol{\Phi}_{d}^{+}(0):=\lim _{t \searrow 0} \boldsymbol{\Phi}_{d}^{\prime}(t) .
$$

We employ this result to prove the following claim, which establishes the Cameron-Martin formula for the more general drift $\tilde{b}$.

Proposition 3. Let $\Sigma \in \mathrm{WIS}_{d}\left(\Sigma_{0}, \tilde{b}, 0, I_{d}\right)$. Then

$$
\mathbb{E}\left[\exp \left\{-\frac{1}{2} \operatorname{tr}\left[w \Sigma_{t}+\int_{0}^{t} v \Sigma_{s} \mathrm{~d} s\right]\right\}\right]=\exp \left\{-\phi(t)-\operatorname{tr}\left[\psi(t) \Sigma_{0}\right]\right\}
$$

where

$$
\psi(t)=-\frac{\sqrt{v} k(t)}{2}, \quad \phi(t)=\frac{1}{2} \operatorname{tr}\left[\tilde{b} \log \left(\sqrt{v}^{-1}(\sqrt{v} \cosh (\sqrt{v} t)+w \sinh (\sqrt{v} t))\right)\right]
$$

and $k(t)$ is given by

$$
k(t)=-(\sqrt{v} \cosh (\sqrt{v} t)+w \sinh (\sqrt{v} t))^{-1}(\sqrt{v} \sinh (\sqrt{v} t)+w \cosh (\sqrt{v} t)) .
$$

Proof. Let us first assume that $\tilde{b}=\alpha I_{d}$. An application of Proposition 2 allows us to claim that

$$
\begin{aligned}
& \mathbb{E}\left[\exp \left\{-\frac{1}{2} \operatorname{tr}\left[w \Sigma_{t}+\int_{0}^{t} v \Sigma_{s} \mathrm{~d} s\right]\right\}\right] \\
& =\operatorname{det}(\cosh (\sqrt{v} t)+\sinh (\sqrt{v} t) k(t))^{\alpha / 2} \exp \left\{\frac{1}{2} \operatorname{tr}\left[\Sigma_{0} \sqrt{v} k(t)\right]\right\}
\end{aligned}
$$

where $k(t)$ is given by

$$
k(t)=-(\sqrt{v} \cosh (\sqrt{v} t)+w \sinh (\sqrt{v} t))^{-1}(\sqrt{v} \sinh (\sqrt{v} t)+w \cosh (\sqrt{v} t)) .
$$


A direct inspection of (8) allows us to recognize the functions $\phi$ and $\psi$ in this setting. For $\psi$, we have

$$
\psi(t)=-\frac{\sqrt{v} k(t)}{2}
$$

which is independent of $\tilde{b}$. The corresponding system of matrix Riccati ODEs is

$$
\begin{array}{cc}
\frac{\mathrm{d} \psi}{\mathrm{d} t}=-2 \psi \psi+v, & \psi(0)=w, \\
\frac{\mathrm{d} \phi}{\mathrm{d} t}=\operatorname{tr}[\tilde{b} \psi(t)], & \phi(0)=0 .
\end{array}
$$

Given the solution for $\psi$, we can determine an alternative formulation for $\phi$ upon integration. This alternative formulation encompasses the more general constant drift too. We show the calculation in detail:

$$
\frac{\mathrm{d} \phi}{\mathrm{d} t}=\operatorname{tr}[\tilde{b} \psi(t)]=\operatorname{tr}\left[\tilde{b}\left(-\frac{\sqrt{v} k(t)}{2}\right)\right]
$$

Integrating the ODE yields

$$
\phi(t)=-\frac{1}{2} \operatorname{tr}\left[\tilde{b} \sqrt{v} \int_{0}^{t} k(s) \mathrm{d} s\right] .
$$

We concentrate on the integral appearing in the second term:

$$
\int_{0}^{t} k(s) \mathrm{d} s=\int_{0}^{t}-(\sqrt{v} \cosh (\sqrt{v} s)+w \sinh (\sqrt{v} s))^{-1}(\sqrt{v} \sinh (\sqrt{v} s)+w \cosh (\sqrt{v} s)) \mathrm{d} s .
$$

Define $f(s)=\sqrt{v} \cosh (\sqrt{v} s)+w \sinh (\sqrt{v} s)$, which upon differentiation yields

$$
\frac{\mathrm{d} f}{\mathrm{~d} s}=(\sqrt{v} \sinh (\sqrt{v} s)+w \cosh (\sqrt{v} s)) \sqrt{v}
$$

hence, we can write

$$
\begin{aligned}
\phi(t) & =\frac{1}{2} \operatorname{tr}[\tilde{b}(\log (\sqrt{v} \cosh (\sqrt{v} t)+w \sinh (\sqrt{v} t))-\log (\sqrt{v}))] \\
& =\frac{1}{2} \operatorname{tr}\left[\tilde{b} \log \left(\sqrt{v}^{-1}(\sqrt{v} \cosh (\sqrt{v} t)+w \sinh (\sqrt{v} t))\right)\right] .
\end{aligned}
$$

2.3.1. Invariance under transformations. We define the transformation $S_{t}=Q^{\top} \Sigma_{t} Q$, which is governed by the SDE

$$
\mathrm{d} S_{t}=\sqrt{S_{t}} \mathrm{~d} \tilde{B}_{t} Q+Q^{\top} \mathrm{d} \tilde{B}_{t}^{\top} \sqrt{S_{t}}+b \mathrm{~d} t, \quad b=Q^{\top} \tilde{b} Q,
$$

where the process $\tilde{B}=\left(\tilde{B}_{t}\right)_{t \geq 0}$ defined by $\mathrm{d} \tilde{B}_{t}={\sqrt{S_{t}}}^{-1} Q^{\top} \sqrt{\Sigma} \mathrm{d} \hat{B}_{t}$ is easily proved to be a Brownian motion under $\tilde{\mathbb{P}}$.

From [5] we know how to extend the Cameron-Martin formula: the Laplace transform of the process $S$ may be computed as

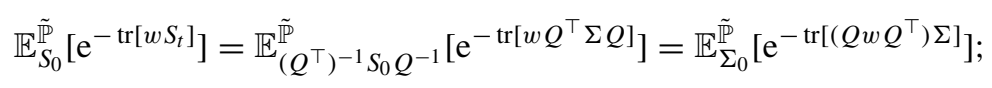

hence, we can compute the Cameron-Martin formula for the process $S$ using the arguments $Q w Q^{\top}$ and $Q v Q^{\top}$.

2.3.2. Inclusion of the drift-Girsanov transformation. The final step consists in introducing a measure change from $\tilde{\mathbb{P}}$, where the process has no mean reversion, to the measure $\mathbb{P}$ that will allow us to consider the general process governed by the dynamics in (1). We now define a 
matrix Brownian motion under the probability measure $\mathbb{P}$ as

$$
B_{t}=\tilde{B}_{t}-\int_{0}^{t} \sqrt{S_{S}} M^{\top} Q^{-1} \mathrm{~d} s=\tilde{B}_{t}-\int_{0}^{t} H_{s} \mathrm{~d} s
$$

for $H_{s}=\sqrt{S_{S}} M^{\top} Q^{-1}$. The Girsanov transformation is given by the following stochastic exponential (see, e.g. [17]):

$$
\begin{aligned}
\left.\frac{\partial \mathbb{P}}{\partial \tilde{\mathbb{P}}}\right|_{\mathcal{F}_{t}} & =\exp \left\{\int_{0}^{t} \operatorname{tr}\left[H^{\top} \mathrm{d} \tilde{B}_{s}\right]-\frac{1}{2} \int_{0}^{t} \operatorname{tr}\left[H H^{\top}\right] \mathrm{d} s\right\} \\
& =\exp \left\{\int_{0}^{t} \operatorname{tr}\left[\left(Q^{-1}\right)^{\top} M \sqrt{S_{s}} \mathrm{~d} \tilde{B}_{s}\right]-\frac{1}{2} \int_{0}^{t} \operatorname{tr}\left[S_{S} M^{\top} Q^{-1}\left(Q^{-1}\right)^{\top} M\right] \mathrm{d} s\right\} .
\end{aligned}
$$

We concentrate on the stochastic integral term, which, under the parametric restriction (5), can be expressed as

$$
\frac{1}{2} \int_{0}^{t} \operatorname{tr}\left[\left(Q^{\top} Q\right)^{-1} M\left(\sqrt{S_{s}} \mathrm{~d} \tilde{B}_{s} Q+Q^{\top} \mathrm{d} \tilde{B}_{s}^{\top} \sqrt{S_{s}}\right)\right]=\frac{1}{2} \int_{0}^{t} \operatorname{tr}\left[\left(Q^{\top} Q\right)^{-1} M\left(\mathrm{~d} S_{s}-b \mathrm{~d} s\right)\right] .
$$

In summary, the stochastic exponential may be written as

$$
\left.\frac{\partial \mathbb{P}}{\partial \tilde{\mathbb{P}}}\right|_{\mathcal{F}_{t}}=\exp \left\{\operatorname{tr}\left[\frac{\left(Q^{\top} Q\right)^{-1} M}{2}\left(S_{t}-S_{0}-b t\right)\right]-\frac{1}{2} \int_{0}^{t} \operatorname{tr}\left[S_{s} M^{\top} Q^{-1}\left(Q^{-1}\right)^{\top} M\right] \mathrm{d} s\right\} .
$$

Under the assumption that $b \succeq(d+1) Q^{\top} Q$ (which is a sufficient condition ensuring that the process does not hit the boundary of the cone $S_{d}^{+}$; see Corrolary 3.2 of [32]), using the same arguments as in [30] shows that the stochastic exponential is a true martingale.

2.3.3. Derivation of the matrix Cameron-Martin formula. We consider the process under $\mathbb{P}$ :

$$
\mathrm{d} S_{t}=\sqrt{S_{t}} \mathrm{~d} B_{t} Q+Q^{\top} \mathrm{d} B_{t}^{\top} \sqrt{S_{t}}+\left(M S_{t}+S_{t} M^{\top}+b\right) \mathrm{d} t .
$$

Recall that, under $\tilde{\mathbb{P}}$, we have

$$
\mathrm{d} S_{t}=\sqrt{S_{t}} \mathrm{~d} \tilde{B}_{t} Q+Q^{\top} \mathrm{d} \tilde{B}_{t}^{\top} \sqrt{S_{t}}+b \mathrm{~d} t .
$$

Then $\Sigma_{t}=\left(Q^{-1}\right)^{\top} S_{t} Q^{-1}$ solves

$$
\mathrm{d} \Sigma_{t}=\sqrt{\Sigma_{t}} \mathrm{~d} \hat{B}_{t}+\mathrm{d} \hat{B}_{t}^{\top} \sqrt{\Sigma_{t}}+\tilde{b} \mathrm{~d} t
$$

We are now ready to apply the change of measure along the following steps:

$$
\begin{aligned}
\mathbb{E}_{S_{0}}^{\mathbb{P}}\left[\exp \left\{-\frac{1}{2} \operatorname{tr}\left[w S_{t}+\int_{0}^{t} v S_{s} \mathrm{~d} s\right]\right\}\right] \\
=\mathbb{E}_{S_{0}}^{\tilde{\mathbb{P}}}\left[\operatorname { e x p } \left\{-\frac{1}{2} \operatorname{tr}\left[w S_{t}+\int_{0}^{t} v S_{s} \mathrm{~d} s\right]+\operatorname{tr}\left[\frac{\left(Q^{\top} Q\right)^{-1} M}{2}\left(S_{t}-S_{0}-b t\right)\right]\right.\right. \\
\left.\left.\quad-\frac{1}{2} \int_{0}^{t} \operatorname{tr}\left[S_{s} M^{\top} Q^{-1}\left(Q^{-1}\right)^{\top} M\right] \mathrm{d} s\right\}\right] \\
=\exp \left\{-\operatorname{tr}\left[\frac{\left(Q^{\top} Q\right)^{-1} M}{2}\left(S_{0}+b t\right)\right]\right\} \\
\times \mathbb{E}_{S_{0}}^{\tilde{\mathbb{P}}}\left[\exp \left\{-\frac{1}{2} \operatorname{tr}\left[\left(w-\left(Q^{\top} Q\right)^{-1} M\right) S_{t}+\int_{0}^{t}\left(v+M^{\top} Q^{-1}\left(Q^{-1}\right)^{\top} M\right) S_{s} \mathrm{~d} s\right]\right\}\right] .
\end{aligned}
$$


But $S_{t}=Q^{\top} \Sigma_{t} Q$. Then

$$
\begin{aligned}
\mathbb{E}_{S_{0}}^{\mathbb{P}}\left[\exp \left\{-\frac{1}{2} \operatorname{tr}\left[w S_{t}+\int_{0}^{t} v S_{s} \mathrm{~d} s\right]\right\}\right] \\
=\exp \left\{-\operatorname{tr}\left[\frac{\left(Q^{\top} Q\right)^{-1} M}{2}\left(S_{0}+b t\right)\right]\right\} \\
\times \mathbb{E}_{\left(Q^{\top}\right)^{-1} S_{0} Q^{-1}\left[\operatorname { e x p } \left\{-\frac{1}{2} \operatorname{tr}\left[Q\left(w-\left(Q^{\top} Q\right)^{-1} M\right) Q^{\top} \Sigma_{t}\right.\right.\right.} \\
\left.\left.\left.\quad+\int_{0}^{t} Q\left(v+M^{\top} Q^{-1}\left(Q^{-1}\right)^{\top} M\right) Q^{\top} \Sigma_{s} \mathrm{~d} s\right]\right\}\right] .
\end{aligned}
$$

The expectation may be computed via a direct application of (8), and after some standard algebra we obtain the result of Theorem 1, with the obvious substitutions $v \rightarrow 2 v$ and $w \rightarrow 2 w$.

2.3.4. Strip of regularity for $\mathfrak{D}_{t}$. Here we show that conditions (6) and (7) imply the boundedness of the Laplace transform for all $t \geq 0$. By Theorem 3.7 of [38] we know that the Laplace transform exists till the explosion time of the solution of the corresponding Riccati ODE. Knowing the explicit solution of such an ODE, a sufficient condition for nonexplosion is that, for all $t \geq 0$,

$$
h(t)=\sqrt{\bar{v}} \cosh (\sqrt{\bar{v}} t)+\bar{w} \sinh (\sqrt{\bar{v}} t) \in \mathrm{GL}_{d} .
$$

As $\bar{v}$ appears in a square root, it must be that $\bar{v} \succeq 0$. However, the inequality must be strict due to $h(0) \in \mathrm{GL}_{d}$. Then $\bar{v} \succ 0$, i.e. condition (6). Now let us rewrite $h(t)$ as

$$
h(t)=\sqrt{\bar{v}} \frac{\mathrm{e}^{\sqrt{\bar{v}} t}+\mathrm{e}^{-\sqrt{\bar{v}} t}}{2}+\bar{w} \frac{\mathrm{e}^{\sqrt{\bar{v}} t}-\mathrm{e}^{-\sqrt{\bar{v}} t}}{2}=\sqrt{\bar{v}} \mathrm{e}^{-\sqrt{\bar{v}} t}+\frac{1}{2}(\sqrt{\bar{v}}+\bar{w})\left(\mathrm{e}^{\sqrt{\bar{v}} t}-\mathrm{e}^{-\sqrt{\bar{v}} t}\right) .
$$

For $\bar{v} \succ 0$, both $\mathrm{e}^{-\sqrt{\bar{v}} t}$ and $\mathrm{e}^{\sqrt{\bar{v}} t}-\mathrm{e}^{-\sqrt{\bar{v}} t}$ belong to $S_{d}^{+}$for all $t \geq 0$. Then $h(t) \in \mathrm{GL}_{d}$ if $\sqrt{\bar{v}}+\bar{w} \succeq 0$, which is condition (7).

\section{Alternative existing methods}

\subsection{Variation-of-constants method}

The variation-of-constants method represents the first solution provided in the literature for the solution of the matrix ODEs (3)-(4) (see, e.g. [21], [22], and [23]), and, despite its theoretical simplicity, it turns out to be very time consuming, as we will show later in the numerical exercise. This is also equivalent to the procedure followed by Ahdida and Alfonsi [1] and Mayerhofer [31], who found the Laplace transform of the Wishart process alone (i.e. corresponding to $v=0$ in (2)). The proof of the following proposition is standard and is thus omitted.

Proposition 4. The solutions for $\psi(t)$ and $\phi(t)$ in Proposition 1 are given by

$$
\begin{aligned}
\psi(t)= & \psi^{\prime}+\mathrm{e}^{\left(M^{\top}-2 \psi^{\prime} Q^{\top} Q\right) t} \\
& \times\left[\left(w-\psi^{\prime}\right)^{-1}+2 \int_{0}^{t} \mathrm{e}^{\left(M-2 Q^{\top} Q \psi^{\prime}\right) s} Q^{\top} Q \mathrm{e}^{\left(M^{\top}-2 \psi^{\prime} Q^{\top} Q\right) s} \mathrm{~d} s\right]^{-1} \\
& \times \mathrm{e}^{\left(M-2 Q^{\top} Q \psi^{\prime}\right) t} \\
\phi(t)= & \operatorname{tr}\left[\alpha Q^{\top} Q \int_{0}^{t} \psi(s) \mathrm{d} s\right]
\end{aligned}
$$


where $\psi^{\prime}$ is a symmetric solution to the following algebraic Riccati equation:

$$
\psi^{\prime} M+M^{\top} \psi^{\prime}-2 \psi^{\prime} Q^{\top} Q \psi^{\prime}+v=0
$$

\subsection{Linearization of the matrix Riccati ODE}

The second approach we consider is that proposed by Grasselli and Tebaldi [24], who used the Radon lemma in order to linearize the matrix Riccati ODE (3) (see also [2], [29], and [39]).

Proposition 5. ([24].) The functions $\psi(t)$ and $\phi(t)$ in Proposition 1 are given by

$$
\begin{aligned}
& \psi(t)=\left(w \psi_{12}(t)+\psi_{22}(t)\right)^{-1}\left(w \psi_{11}(t)+\psi_{21}(t)\right), \\
& \phi(t)=\frac{\alpha}{2} \operatorname{tr}\left[\log \left(w \psi_{12}(t)+\psi_{22}(t)\right)+M^{\top} t\right],
\end{aligned}
$$

where

$$
\left(\begin{array}{ll}
\psi_{11}(t) & \psi_{12}(t) \\
\psi_{21}(t) & \psi_{22}(t)
\end{array}\right)=\exp \left\{t\left(\begin{array}{cc}
M & 2 Q^{\top} Q \\
v & -M^{\top}
\end{array}\right)\right\}
$$

\subsection{Runge-Kutta method}

The Runge-Kutta method is a classical approach for the numerical solution of ODEs. For a detailed treatment, see, e.g. [36]. If we want to solve numerically the system of equations (3) and (4), the most commonly used Runge-Kutta scheme is the fourth-order scheme, i.e.

$$
\begin{aligned}
\psi\left(t_{n+1}\right) & =\psi\left(t_{n}\right)+\frac{1}{6} h\left(k_{1}+2 k_{2}+2 k_{3}+k_{4}\right), \\
t_{n+1} & =t_{n}+h, \\
k_{1} & =g\left(t_{n}, \psi\left(t_{n}\right)\right), \\
k_{2} & =g\left(t_{n}+\frac{1}{2} h, \psi\left(t_{n}\right)+\frac{1}{2} h k_{1}\right), \\
k_{3} & =g\left(t_{n}+\frac{1}{2} h, \psi\left(t_{n}\right)+\frac{1}{2} h k_{2}\right), \\
k_{4} & =g\left(t_{n}+h, \psi\left(t_{n}\right)+h k_{3}\right),
\end{aligned}
$$

where the function $g$ is given by

$$
g\left(t_{n}, \psi\left(t_{n}\right)\right)=g\left(\psi\left(t_{n}\right)\right)=\psi\left(t_{n}\right) M+M^{\top} \psi\left(t_{n}\right)-2 \psi\left(t_{n}\right) Q^{\top} Q \psi\left(t_{n}\right)+v .
$$

\subsection{Comparison of the methods}

A formal numerical analysis of the various methods is beyond the scope of this paper. Anyhow, we would like to stress some points about the execution times, which we believe are sufficient to highlight the importance of our new methodology. Despite its importance in the academic literature, it will turn out that the variation-of-constants method is not suitable for applications, in particular in a calibration setting.

We first compare the results of the four different methods. We consider different time horizons $t \in[0,3.0]$ and use the following values for the parameters: $\alpha=3$,

$$
\begin{array}{rlr}
S_{0}=\left(\begin{array}{ll}
0.0120 & 0.0010 \\
0.0010 & 0.0030
\end{array}\right), & Q=\left(\begin{array}{cc}
0.141421356237310 & -0.070710678118655 \\
0 & 0.070710678118655
\end{array}\right), \\
M=\left(\begin{array}{ll}
-0.02 & -0.02 \\
-0.01 & -0.02
\end{array}\right), & v=\left(\begin{array}{ll}
0.1000 & 0.0400 \\
0.0400 & 0.1000
\end{array}\right), \quad w=\left(\begin{array}{ll}
0.1100 & 0.0300 \\
0.0300 & 0.1100
\end{array}\right) .
\end{array}
$$


The value for $Q$ was obtained along the following steps: given a matrix $A \in S_{d}^{+}$such that $A M=M^{\top} A$, we compute its inverse and let $Q$ be obtained from a Cholesky factorization of this inverted matrix.

In Table 1 we present the values of the moment generating function for different time horizons $t$. The four methods lead to values which are very close to each other, and this constitutes a first test proving that the new methodology produces correct results. Let us now consider another important issue, namely the execution speed. In order to obtain a good degree of precision for the variation-of-constants method, we were forced to employ a fine integration grid. This results in a poor performance of this method in terms of speed. In Figure 1 we compare the time taken by the three analytical methods to calculate the moment generating function. As $t$ gets larger, the execution time for the variation-of-constants method grows exponentially, whereas the time required by the linearization and the new methodology are the

TABLE 1: Computations of the joint moment generating function of the Wishart process and its time integral for different time horizons $\tau$. All four methods are considered. It should be noted that the variation-of-constants method requires a very fine integration grid in order to produce precise values that can be compared with the results of the other methods.

\begin{tabular}{ccccc}
\hline $\begin{array}{c}\text { Time } \\
\text { horizon }\end{array}$ & Linearization & Cameron-Martin & Variation of constants & Runge-Kutta \\
\hline 0.0 & 0.998291461216988 & 0.998291461216988 & 0.998291461216988 & 0.998291461216988 \\
0.1 & 0.997303305375919 & 0.997303305375919 & 0.997306285702955 & 0.997271605593416 \\
0.5 & 0.992740622447456 & 0.992740622447456 & 0.992703104707601 & 0.992583959952442 \\
1.0 & 0.985698139368470 & 0.985698139368470 & 0.985426551402640 & 0.985389882322825 \\
1.5 & 0.977224894409802 & 0.977224894409802 & 0.976522044659620 & 0.976770850175581 \\
2.0 & 0.967388334051965 & 0.967388334051964 & 0.966066486500228 & 0.966794966212865 \\
2.5 & 0.956261597343174 & 0.956261597343174 & 0.954144681472186 & 0.955535938544691 \\
3.0 & 0.943922618087738 & 0.943922618087738 & 0.940848141282233 & 0.943072180564490 \\
\hline
\end{tabular}

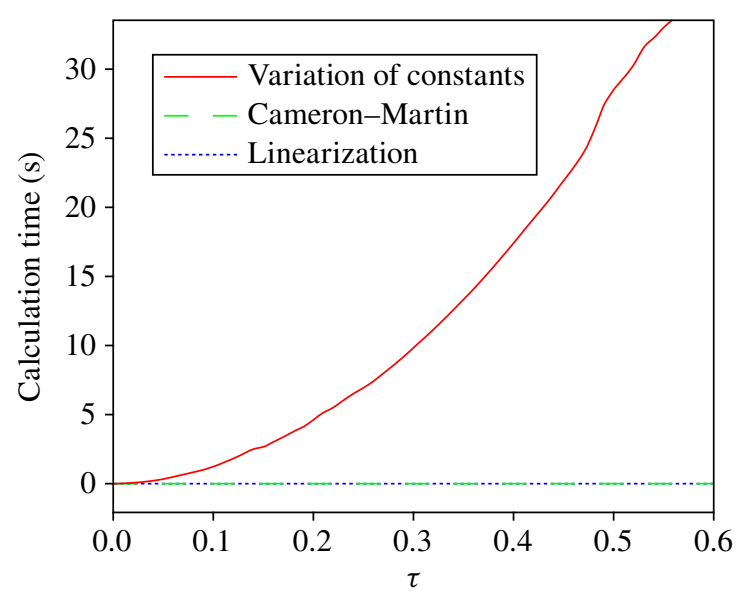

Figure 1: The calculation times of the three analytical methods (variation of constants, Cameron-Martin, and linearization) to compute the joint moment generating function of the Wishart process and its time integral for different time horizons. Note that the variation-of-constants method is inefficient even for quite short maturities. 
TABLE 2: In this table we do not include the results for the variation-of-constants method. This allows us to look at a longer time horizon and, therefore, appreciate the precision of the new methodology.

\begin{tabular}{cccc}
\hline $\begin{array}{c}\text { Time } \\
\text { horizon }\end{array}$ & Linearization & Cameron-Martin & Runge-Kutta \\
\hline 0.0 & 0.998291461216988 & 0.998291461216988 & 0.998291461216988 \\
0.1 & 0.997303305375919 & 0.997303305375919 & 0.997271605593416 \\
0.5 & 0.992740622447456 & 0.992740622447456 & 0.992583959952442 \\
1.0 & 0.985698139368470 & 0.985698139368470 & 0.985389882322825 \\
2.0 & 0.967388334051965 & 0.967388334051964 & 0.966794966212865 \\
3.0 & 0.943922618087738 & 0.943922618087738 & 0.943072180564490 \\
4.0 & 0.915938197508059 & 0.915938197508059 & 0.914862207389661 \\
5.0 & 0.884120166104796 & 0.884120166104796 & 0.882852196560219 \\
10.0 & 0.691634000576684 & 0.691634000576684 & 0.689897813632122 \\
100.0 & 0.000001636282753 & 0.000001636282753 & 0.000001629036716 \\
\hline
\end{tabular}

same. The Runge-Kutta method is a numerical solution to the problem, so the real competitors of our methodology are the variation-of-constants and the linearization methods.

Finally, we compared the linearization of the Riccati ODE to the new methodology. In terms of precision and execution speed the two methodologies seem to provide the same performance, up to the fourteenth digit. This shows that, under the parametric restriction of Theorem 1, our methodology represents a valid alternative. The results are illustrated in Table 2 up to the maturity $t=100$.

\section{Applications}

\subsection{Pricing of derivatives}

The knowledge of the functional form of the Laplace transform represents an important tool for the application of a stochastic model in mathematical finance. In the following we will provide two examples of asset pricing models whose Laplace transform is of exponentially affine form and such that our previous results may be applied. In the first example we consider the model proposed in [16] which describes the evolution of a single asset, whose instantaneous volatility is modelled by means of a Wishart process. In the second example we consider the model introduced in [15], in which the evolution of a vector of assets is described by a vectorvalued SDE where the Wishart process models the instantaneous variance-covariance matrix of the assets.

4.1.1. A stochastic volatility model. In this subsection we consider the model proposed in [16] and we derive the explicit Laplace transform of the log-price using our new methodology. As a starting point, we report the dynamics defining the model:

$$
\begin{aligned}
\frac{\mathrm{d} X_{t}}{X_{t}} & =\operatorname{tr}\left[\sqrt{S_{t}}\left(\mathrm{~d} W_{t} R^{\top}+\mathrm{d} B_{t} \sqrt{I_{d}-R R^{\top}}\right)\right], \\
\mathrm{d} S_{t} & =\left(\alpha Q^{\top} Q+M S_{t}+S_{t} M^{\top}\right) \mathrm{d} t+\sqrt{S_{t}} \mathrm{~d} W_{t} Q+Q^{\top} \mathrm{d} W_{t}^{\top} \sqrt{S_{t}} .
\end{aligned}
$$

Here $X_{t}$ denotes the price of the underlying asset, the Wishart process acts as a multifactor source of stochastic volatility, $W$ and $B$ are independent matrix Brownian motions, and the matrix $R$ parametrizes all possible correlation structures preserving the affinity. This model is a generalization of the (multi-)Heston model, see [9] and [25], and it offers a very rich structure 
for the modelization of stochastic volatilities as the factors governing the instantaneous variance are nontrivially correlated. It is easy to see that the $\log$-price $Y$ is given as

$$
\mathrm{d} Y=-\frac{1}{2} \operatorname{tr}\left[S_{t}\right] \mathrm{d} t+\operatorname{tr}\left[\sqrt{S_{t}}\left(\mathrm{~d} W_{t} R^{\top}+\mathrm{d} B_{t} \sqrt{I_{d}-R R^{\top}}\right)\right] .
$$

We are interested in the Laplace transform of the log-price, i.e.

$$
\varphi_{t}(\tau,-\omega)=\mathbb{E}\left[\mathrm{e}^{-\omega Y_{T}} \mid \mathcal{F}_{t}\right], \quad \tau:=T-t .
$$

This expectation satisfies a backward Kolmogorov equation; see [16] for a detailed derivation. Since the process $S=\left(S_{t}\right)_{0 \leq t \leq T}$ is affine, we guess that the solution is of the form

$$
\varphi_{t}(\tau,-\omega)=\exp \left\{-\omega \ln X_{t}-\phi(\tau)-\operatorname{tr}\left[\psi(\tau) S_{t}\right]\right\}
$$

Upon substitution into the PDE, we obtain the system of ODEs

$$
\begin{gathered}
\frac{\mathrm{d} \psi}{\mathrm{d} \tau}=\psi\left(M-\omega Q^{\top} R^{\top}\right)+\left(M^{\top}-\omega R Q\right) \psi-2 \psi Q^{\top} Q \psi-\frac{\omega^{2}+\omega}{2} I_{d}, \quad \psi(0)=0, \\
\frac{\mathrm{d} \phi}{\mathrm{d} \tau}=\operatorname{tr}\left[\alpha Q^{\top} Q \psi(\tau)\right], \quad \phi(0)=0 .
\end{gathered}
$$

If we look at the first ODE, we recognize the same structure as in (3): instead of $M$ and $v$ we respectively have $M-\omega Q^{\top} R^{\top}$ and $-\frac{1}{2}\left(\omega^{2}+\omega\right) I_{d}$. This means that we can rewrite the solution for $\psi$ as

$$
\begin{aligned}
\psi(\tau) & =\frac{\left(Q^{\top} Q\right)^{-1}\left(M-\omega Q^{\top} R^{\top}\right)}{2}-\frac{Q^{-1} \sqrt{\bar{v}} k\left(Q^{\top}\right)^{-1}}{2}, \\
\phi(\tau) & =-\frac{\alpha}{2} \log \left(\operatorname{det}\left(\mathrm{e}^{-\left(M-\omega Q^{\top} R^{\top}\right) \tau}(\cosh (\sqrt{\bar{v} \tau})+\sinh (\sqrt{\bar{v} \tau}) k)\right)\right), \\
\bar{v} & =Q\left(2\left(-\frac{\omega^{2}+\omega}{2} I_{d}\right)+\left(M^{\top}-\omega R Q\right) Q^{-1}\left(Q^{\top}\right)^{-1}\left(M-\omega Q^{\top} R^{\top}\right)\right) Q^{\top}, \\
\bar{w} & =Q\left(-\left(Q^{\top} Q\right)^{-1}\left(M-\omega Q^{\top} R^{\top}\right)\right) Q^{\top}, \\
k & =-(\sqrt{\bar{v}} \cosh (\sqrt{\bar{v}} \tau)+\bar{w} \sinh (\sqrt{\bar{v}} \tau))^{-1}(\sqrt{\bar{v}} \sinh (\sqrt{\bar{v}} \tau)+\bar{w} \cosh (\sqrt{\bar{v}} \tau)) .
\end{aligned}
$$

Condition (5) in this setting has the form

$$
\left(M-\omega Q^{\top} R^{\top}\right)^{\top}\left(Q^{\top} Q\right)^{-1}=\left(Q^{\top} Q\right)^{-1}\left(M-\omega Q^{\top} R^{\top}\right) .
$$

For fixed $\omega$, we can express the condition above via the following system:

$$
M^{\top}\left(Q^{\top} Q\right)^{-1}=\left(Q^{\top} Q\right)^{-1} M, \quad R Q\left(Q^{\top} Q\right)^{-1}=\left(Q^{\top} Q\right)^{-1} Q^{\top} R^{\top} .
$$

4.1.2. A stochastic correlation model. In this subsection we consider the model introduced in [15]. This model belongs to the class of multivariate affine volatility models, for which many interesting theoretical results have been presented in [10]. In this framework we consider a vector of prices together with a stochastic variance-covariance matrix, i.e.

$$
\begin{aligned}
\mathrm{d} X_{t} & =\operatorname{diag}\left(X_{t}\right) \sqrt{S_{t}}\left(\mathrm{~d} W_{t} \rho+\sqrt{1-\rho^{\top} \rho} \mathrm{d} B_{t}\right), \\
\mathrm{d} S_{t} & =\left(\alpha Q^{\top} Q+M S_{t}+S_{t} M^{\top}\right) \mathrm{d} t+\sqrt{S_{t}} \mathrm{~d} W_{t} Q+Q^{\top} \mathrm{d} W_{t}^{\top} \sqrt{S_{t}},
\end{aligned}
$$


where now the vector Brownian motion $Z=W_{t} \rho+\sqrt{1-\rho^{\top} \rho} B_{t}$ is correlated with the matrix Brownian motion $W$ through the correlation vector $\rho$. Using the same arguments as before, we compute the joint conditional Laplace transform of the vector of the $\log$-prices $Y_{T}=\log \left(X_{T}\right)$ as

$$
\varphi_{t}(\tau,-\omega)=\mathbb{E}\left[\mathrm{e}^{-\omega^{\top} Y_{T}} \mid \mathcal{F}_{t}\right], \quad \tau:=T-t .
$$

The affine property allows us to write the associated system of matrix Riccati ODEs (see [15] for more details) as

$$
\begin{aligned}
\frac{\mathrm{d} \psi}{\mathrm{d} \tau} & =\psi\left(M-Q^{\top} \rho \omega^{\top}\right)+\left(M^{\top}-\omega \rho^{\top} Q\right) \psi-2 \psi Q^{\top} Q \psi-\frac{1}{2}\left(\sum_{i=1}^{d} \omega_{i} e_{i i}+\omega^{\top} \omega\right) I_{d}, \\
\psi(0) & =0,
\end{aligned}
$$

and

$$
\frac{\mathrm{d} \phi}{\mathrm{d} \tau}=\operatorname{tr}\left[\alpha Q^{\top} Q \psi(\tau)\right], \quad \phi(0)=0 .
$$

We recognize the same structure as in (4) and (3) where instead of $M$ and $v$, we now have $M-Q^{\top} \rho \omega^{\top}$ and $-\frac{1}{2}\left(\sum_{i=1}^{d} \omega_{i} e_{i i}+\omega^{\top} \omega\right) I_{d}$, respectively. Consequently, we can compute the solution as

$$
\begin{aligned}
\psi(\tau) & =\frac{\left(Q^{\top} Q\right)^{-1}\left(M-Q^{\top} \rho \omega^{\top}\right)}{2}-\frac{Q^{-1} \sqrt{\bar{v}} k\left(Q^{\top}\right)^{-1}}{2} \\
\phi(\tau) & =-\frac{\alpha}{2} \log \left(\operatorname{det}\left(\mathrm{e}^{-\left(M-Q^{\top} \rho \omega^{\top}\right) \tau}(\cosh (\sqrt{\bar{v} \tau})+\sinh (\sqrt{\bar{v} \tau}) k)\right)\right) \\
\bar{v} & =Q\left(2\left(-\frac{1}{2}\left(\sum_{i=1}^{d} \omega_{i} e_{i i}+\omega^{\top} \omega\right) I_{d}\right)+\left(M^{\top}-\omega \rho Q\right) Q^{-1}\left(Q^{\top}\right)^{-1}\left(M-Q^{\top} \rho \omega^{\top}\right)\right) Q^{\top}, \\
\bar{w} & =Q\left(-\left(Q^{\top} Q\right)^{-1}\left(M-Q^{\top} \rho \omega^{\top}\right)\right) Q^{\top}, \\
k & =-(\sqrt{\bar{v}} \cosh (\sqrt{\bar{v}} \tau)+\bar{w} \sinh (\sqrt{\bar{v}} \tau))^{-1}(\sqrt{\bar{v}} \sinh (\sqrt{\bar{v}} \tau)+\bar{w} \cosh (\sqrt{\bar{v}} \tau)) .
\end{aligned}
$$

Condition (5) is rephrased in this setting as

$$
\left(M^{\top}-\omega \rho^{\top} Q\right)\left(Q^{\top} Q\right)^{-1}=\left(Q^{\top} Q\right)^{-1}\left(M-Q^{\top} \rho \omega^{\top}\right),
$$

which may be expressed as

$$
M^{\top}\left(Q^{\top} Q\right)^{-1}=\left(Q^{\top} Q\right)^{-1} M, \quad \omega \rho^{\top}\left(Q^{\top}\right)^{-1}=Q^{-1} \rho \omega^{\top} .
$$

This means that the two products have to be symmetric matrices.

4.1.3. A short-rate model. Our methodology for the computation of the Laplace transform may be directly employed to provide a closed-form formula for the price of zero-coupon bonds when the short rate is driven by a Wishart process. The Wishart short-rate model has been studied in [6], [8], [19], [21], and [24]. The short rate is modelled as

$$
r_{t}=a+\operatorname{tr}\left[v S_{t}\right]
$$

where $a \in \mathbb{R}_{\geq 0}, v$ is a symmetric positive-definite matrix, and $S=\left(S_{t}\right)_{t \geq 0}$ is the Wishart process. Standard arbitrage arguments allow us to claim that the price of a zero-coupon bond 
at time $t$ with time to maturity $\tau:=T-t$, denoted by $P_{t}(\tau)$, is given by the expectation

$$
P_{t}(\tau):=\mathbb{E}\left[\exp \left\{-\int_{t}^{T}\left(a+\operatorname{tr}\left[v X_{u}\right]\right) \mathrm{d} u\right\} \mid \mathcal{F}_{t}\right]=\exp \left\{-\phi(\tau)-\operatorname{tr}\left[\psi(\tau) X_{t}\right]\right\},
$$

where the associated ODEs are

$$
\frac{\partial \phi}{\partial \tau}=\operatorname{tr}\left[\alpha Q^{\top} Q \psi(\tau)\right]+a, \quad \phi(0)=0,
$$

and

$$
\frac{\partial \psi}{\partial \tau}=\psi(\tau) M+M^{\top} \psi(\tau)-2 \psi(\tau) Q^{\top} Q \psi(\tau)+v, \quad \psi(0)=0 .
$$

We can again employ the Cameron-Martin formula and write the solution to the system as

$$
\begin{aligned}
& \psi(\tau)=\frac{\left(Q^{\top} Q\right)^{-1} M}{2}-\frac{Q^{-1} \sqrt{\bar{v}} k\left(Q^{\top}\right)^{-1}}{2}, \\
& \phi(\tau)=-\frac{\alpha}{2} \log \left(\operatorname{det}\left(\mathrm{e}^{-M \tau}(\cosh (\sqrt{\bar{v}} \tau)+\sinh (\sqrt{\bar{v}} \tau) k)\right)\right)+a \tau .
\end{aligned}
$$

\subsection{A solution to the algebraic Riccati equation (10)}

As an application of our result of independent interest, we look at the problem of computing a solution to the algebraic Riccati equation (ARE) (10). This equation is well known from control theory and only numerical methods are available for computing its solution. We will construct a solution to the ARE by comparing the solution of the system of differential equations in Proposition 1 obtained according to our new methodology and the variation-of-constant approach. For convenience, rewrite the system of ODEs (3)-(4) as

$$
\frac{\mathrm{d} \psi}{\mathrm{d} t}=\mathcal{R}(\psi), \quad \psi(0)=w, \quad \frac{\mathrm{d} \phi}{\mathrm{d} t}=\mathcal{F}(\psi), \quad \phi(0)=0 .
$$

An ARE is given by

$$
\mathcal{R}\left(\psi^{\prime}\right)=0 .
$$

As before, we denote by $\psi^{\prime}$ a solution to this equation.

Lemma 1. Let $O \in S_{d}^{+}$, and define

$$
\sinh (O \tau)=\frac{\mathrm{e}^{O \tau}-\mathrm{e}^{-O \tau}}{2}, \quad \cosh (O \tau)=\frac{\mathrm{e}^{O \tau}+\mathrm{e}^{-O \tau}}{2}
$$

and

$$
\tanh (O \tau)=(\cosh (O \tau))^{-1} \sinh (O \tau), \quad \operatorname{coth}(O \tau)=(\sinh (O \tau))^{-1} \cosh (O \tau) .
$$

Then

$$
\lim _{\tau \rightarrow \infty} \tanh (O \tau)=\lim _{\tau \rightarrow \infty} \operatorname{coth}(O \tau)=I_{d}
$$

Proof. Let $A \in M_{d}$. If $\operatorname{Re}(\lambda(A))<0$ for all $\lambda \in \sigma(A)$ then it is well known that

$$
\lim _{\tau \rightarrow \infty} \mathrm{e}^{A \tau}=0 \in M_{d \times d}
$$

Consequently, we have

$$
\lim _{\tau \rightarrow \infty} \tanh (O \tau)=\lim _{\tau \rightarrow \infty}\left(I_{d}+\mathrm{e}^{-2 O \tau}\right)^{-1}\left(I_{d}-\mathrm{e}^{-2 O \tau}\right)=I_{d} .
$$

The second equality follows along the same lines. 
Let us recall some well-known results from control theory. We refer the reader to the review article [27]. Let us write $v=C^{\top} C$. We introduce the following notions.

- The pair $(M, Q)$ is said to be stabilizable if there exists a matrix $L$ such that $M+Q L$ is stable, i.e. all the eigenvalues are negative.

- The pair $(C, M)$ is said to be detectable if there exists a matrix $F$ such that $F C+M$ is stable.

We introduce again the matrix $M-2 Q^{\top} Q \psi^{\prime}$ and call it the closed-loop system matrix. A classical result is the following.

Theorem 2. Stabilizability of $(M, Q)$ and detectability of $(C, M)$ is necessary and sufficient for the ARE to have a unique nonnegative solution which makes the closed loop system matrix stable.

Now, looking at the variation-of-constants approach we can prove the next result.

Corollary 1. It holds that

$$
\lim _{\tau \rightarrow \infty} \psi(\tau)=\psi^{\prime}
$$

Proof. Under the assumptions of Theorem 2, we have $\lambda\left(M-2 Q^{\top} Q \psi^{\prime}\right)<0$ for all $\lambda \in$ $\sigma\left(M-2 Q^{\top} Q \psi^{\prime}\right)$; hence, we know that the integral in (9), the solution for $\psi$, is convergent, and moreover, we know that $\mathrm{e}^{\left(M-2 Q^{\top} Q \psi^{\prime}\right) \tau} \searrow 0$ as $\tau \rightarrow \infty$. This completes the proof.

This last corollary tells us that the function $\psi$ tends to a stability point of the Riccati ODE. This allows us to claim that, as $\tau \rightarrow \infty$, we have $\mathcal{R}(\psi(\tau)) \searrow 0$. A nice consequence of this fact is that we are able to provide a new representation for $\psi^{\prime}$, which constitutes another application of the Cameron-Martin approach.

Proposition 6. The value of $\psi^{\prime}$ in Corrolary 1 admits the representation

$$
\psi^{\prime}=\frac{Q^{-1} \sqrt{\bar{v}}\left(Q^{\top}\right)^{-1}}{2}+\frac{\left(Q^{\top} Q\right)^{-1} M}{2} .
$$

Proof. On the basis of Theorem 1, we want to compute

$$
\lim _{\tau \rightarrow \infty}-\frac{Q^{-1} \sqrt{\bar{v}} k(\tau)\left(Q^{\top}\right)^{-1}}{2}+\frac{\left(Q^{\top} Q\right)^{-1} M}{2} .
$$

To perform the computation, it is sufficient to calculate

$$
\begin{aligned}
\lim _{\tau \rightarrow \infty} k(\tau) & =\lim _{\tau \rightarrow \infty}\left\{-(\sqrt{\bar{v}} \cosh (\sqrt{\bar{v}} \tau)+\bar{w} \sinh (\sqrt{\bar{v}} \tau))^{-1}(\sqrt{\bar{v}} \sinh (\sqrt{\bar{v}} \tau)+\bar{w} \cosh (\sqrt{\bar{v}} \tau))\right\} \\
& =\lim _{\tau \rightarrow \infty}\left\{-(\cosh \sqrt{\bar{v}} \tau)^{-1}(\sqrt{\bar{v}}+\bar{w} \tanh \sqrt{\bar{v}} \tau)^{-1}(\sqrt{\bar{v}}+\bar{w} \operatorname{coth} \sqrt{\bar{v}} \tau) \sinh \sqrt{\bar{v}} \tau\right\}
\end{aligned}
$$

From Lemma 1 we know that both tanh and coth tend to $I_{d}$ as $\tau \rightarrow \infty$; hence, we conclude that

$$
\lim _{\tau \rightarrow \infty} k(\tau)=-I_{d}
$$

and so we obtain the final claim:

$$
\lim _{\tau \rightarrow \infty} \psi(\tau)=\frac{Q^{-1} \sqrt{\bar{v}}\left(Q^{\top}\right)^{-1}}{2}+\frac{\left(Q^{\top} Q\right)^{-1} M}{2} .
$$

Finally, from Corrolary 1, we know that $\lim _{\tau \rightarrow \infty} \psi(\tau)=\psi^{\prime}$, completing the proof. 


\section{Conclusions}

In this paper we derived a new explicit formula for the joint Laplace transform of the Wishart process and its time integral based on the original approach of [5]. Our methodology leads to a truly explicit formula that does not involve any additional integration (like the highly time consuming variation-of-constants method) or blocks of matrix exponentials (like the linearization method) at the price of a simple condition on the parameters. We presented some examples of applications in the context of multifactor and multivariate stochastic volatility. Moreover, we provided an explicit solution to the algebraic Riccati ODE that appears in linearquadratic control theory and for which only numerical schemes are available. We also recalled a recent application of our result by Bäuerle and Li [4] in the portfolio optimization setting of [13].

\section{Acknowledgements}

We are indebted to José Da Fonseca, Antoine Jacquier, Kyoung-Kuk Kim, Eberhard Mayerhofer, Eckhard Platen, Wolfgang Runggaldier, and an anonymous referee for helpful suggestions.

\section{References}

[1] Ahdida, A. And Alfonsi, A. (2013). Exact and high-order discretization schemes for Wishart processes and their affine extensions. Ann. Appl. Prob. 23, 1025-1073.

[2] Anderson, B. D. O. And Moore, J. B. (1971). Linear Optimal Control. Prentice-Hall, Englewood Cliffs, NJ.

[3] Barndorff-Nielsen, O. E. and Stelzer, R. (2007). Positive-definite matrix processes of finite variation. Prob. Math. Statist. 27, 3-43.

[4] Bäuerle, N. ANd Li, Z. (2013). Optimal portfolios for financial markets with Wishart volatility. J. Appl. Prob. 50, 1025-1043.

[5] BRU, M.-F. (1991). Wishart processes. J. Theoret. Prob. 4, 725-751.

[6] Buraschi, A., Cieslak, A. and Trojani, F. (2008). Correlation risk and the term structure of interest rates. Working paper. Available at http://www.andreaburaschi.com/research.htm.

[7] Buraschi, A., Porchia, P. And Trojani, F. (2010). Correlation risk and optimal portfolio choice. J. Finance 65, 393-420.

[8] Chiarella, C., Hsiao, C.-Y. and To, T.-D. (2010). Risk premia and Wishart term structure models. Working paper. Available at http://papers.ssrn.com/sol3/papers.cfm?abstract-id=1573184.

[9] Christoffersen, P., Heston, S. And Jacobs, K. (2009). The shape and term structure of the index option smirk: why multifactor stochastic volatility models work so well. Manag. Sci. 55, 1914-1932.

[10] Cuchiero, C. (2011). Affine and polynomial processes. Doctoral Thesis, ETH Zürich.

[11] Cuchiero, C., Filipović, D., Mayerhofer, E. and Teichmann, J. (2011). Affine processes on positive semidefinite matrices. Ann. App. Prob. 21, 397-463.

[12] Da Fonseca, J. and Grasselli, M. (2011). Riding on the smiles. Quant. Finance 11, 1609-1632.

[13] Da Fonseca, J., Grasselli, M. ANd Ielpo, F. (2011). Hedging (co)variance risk with variance swaps. Internat. J. Theoret. Appl. Finance 14, 899-943.

[14] Da Fonseca, J., Grasselli, M. And Ielpo, F. (2014). Estimating the Wishart affine stochastic correlation model using the empirical characteristic function. Stud. Nonlinear Dynam. Econometrics 18, 253-289.

[15] Da Fonseca, J., Grasselli, M. and Tebaldi, C. (2007). Option pricing when correlations are stochastic: an analytical framework. Rev. Derivatives Res. 10, 151-180.

[16] Da Fonseca, J., Grasselli, M. and Tebaldi, C. (2008). A multifactor volatility Heston model. Quant. Finance 8, 591-604.

[17] Donati-Martin, C., Doumerc, Y., Matsumoto, H. and Yor, M. (2004). Some properties of the Wishart processes and a matrix extension of the Hartman-Watson laws. Publ. Res. Inst. Math. Sci. 40, 1385-1412.

[18] Duffie, D., Filipović, D. And Schachermayer, W. (2003). Affine processes and applications in finance. Ann. Appl. Prob. 13, 984-1053.

[19] Gnoatto, A. (2012). The Wishart short rate model. Internat. J. Theoret. Appl. Finance 15, 1250056.

[20] Gourieroux, C. (2006). Continuous time Wishart process for stochastic risk. Econometric Rev. 25, $177-217$.

[21] Gourieroux, C. And Sufana, R. (2003). Wishart quadratic term structure models. Working paper. Available at http://papers.ssrn.com/sol3/papers.cfm?abstract_id=757307. 
[22] Gourieroux, C. and Sufana, R. (2010). Derivative pricing with Wishart multivariate stochastic volatility. $J$. Bus. Econom. Statist. 28, 438-451.

[23] Gourieroux, C., Monfort, A. and Sufana, R. (2010). International money and stock market contingent claims. J. Internat. Money Finance 29, 1727-1751.

[24] Grasselli, M. and Tebaldi, C. (2008). Solvable affine term structure models. Math. Finance 18, $135-153$.

[25] Heston, S. L. (1993). A closed-form solution for options with stochastic volatility with applications to bond and currency options. Rev. Financial Studies 6, 327-343.

[26] KANG, C. AND KANG, W. (2013). Transform formulae for linear functionals of affine processes and their bridges on positive semidefinite matrices. Stoch. Process. Appl. 123, 2419-2445.

[27] KučERA, V. (1973). A review of the matrix Riccati equation. Kybernetika 9, 42-61.

[28] Leiprold, M. And Trojani, F. (2010). Asset pricing with matrix jump diffusions. Working paper. Available at http://papers.ssrn.com/sol3/papers.cfm?abstract_id=1274482.

[29] Levin, J. J. (1959). On the matrix Riccati equation. Proc. Amer. Math. Soc. 10, 519-524.

[30] MaYerhofer, E. (2012). Wishart processes and Wishart distributions: an affine processes point of view. CIMPA lecture notes. Available at http://arxiv.org/abs/1201.6634.

[31] Mayerhofer, E. (2013). On the existence of non-central Wishart distributions. J. Multivariate Anal. 114, 448456.

[32] Mayerhofer, E., Pfaffel, O. and Stelzer, R. (2011). On strong solutions for positive definite jump diffusions. Stoch. Process. Appl. 121, 2072-2086.

[33] Muhle-Karbe, J., Pfaffel, O. and Stelzer, R. (2012). Option pricing in multivariate stochastic volatility models of OU type. SIAM J. Financial Math. 3, 66-94.

[34] Pigorsch, C. ANd Stelzer, R. (2009). On the definition, stationary distribution and second order structure of positive semidefinite Ornstein-Uhlenbeck type processes. Bernoulli 15, 754-773.

[35] Pitman, J. and Yor, M. (1982). A decomposition of Bessel bridges. Z. Wahrscheinlichkeitsth. 59, $425-457$.

[36] Quarteroni, A., Sacco, R. And Saleri, F. (2000). Numerical Mathematics (Texts Appl. Math. 37). Springer, New York.

[37] Revuz, D. AND Yor, M. (1994). Continuous Martingales and Brownian Motion (Fundamental Principles Math. Sci. 293), 2nd edn. Springer, Berlin.

[38] SpreiJ, P. and Veerman, E. (2010). The affine transform formula for affine jump-diffusions with a general closed convex state space. Preprint. Available at http://arxiv.org/abs/1005.1099.

[39] Yong, J. And Zhou, X. Y. (1999). Stochastic Controls: Hamiltonian Systems and HJB Equations. Springer, New York. 